\title{
How well do women recall past contraceptive use? A pilot study
}

\author{
Anja Katharina Guttinger, Zhong Eric Chen
}

\begin{abstract}
Background and methodology Many women use a variety of contraceptive methods during their reproductive lives. Investigating this exposure is one of the most frequently performed epidemiological investigations. Accurate recall of methods used, as well as validating this information, can be difficult. A pilot study compared recalled contraceptive use over 5 years with that documented in the case notes of 30 women.
\end{abstract}

Results $47 \%$ of episodes of method use were accurately recalled to the month of starting method use; this figure rose to $94 \%$ when episodes with disagreement within \pm 12 months were also considered. Similarly, $44 \%$ and $91 \%$ of episodes were accurately recalled to the month and within \pm 12 months of stopping method use, respectively. Accuracy of recall for duration of use followed a similar pattern. $7 \%$ of users were unable to distinguish between use of a combined pill and a progestogen-only pill and one-third of women using an intrauterine contraceptive were unable to distinguish an intrauterine device (IUD) from the intrauterine system (IUS).

Discussion and conclusions Almost all women can recall accurately which contraceptive methods they have used in the past year but are less accurate in respect of exact starting and stopping dates. Some women confuse the combined pill with the progestogen-only pill and others confuse the IUD and the IUS. The findings need to be replicated in other settings and with populations of less well-educated women.

Keywords contraceptive use, interview, prescriber records, recall, validation studies

J Fam Plann Reprod Health Care 2009; 35(3): 173-175

(Accepted 19 January 2009)

\section{Introduction}

Understanding patterns of contraceptive use over time and, more specifically, gathering information on continuation rates and method switching, is essential to developing interventions designed to improve effective contraceptive use and to evaluating their success. Many women use a number of different contraceptive methods over time. Investigating exposure to reproductive medicines, particularly oral contraceptives and hormone replacement therapy, is one of the most frequently performed epidemiological investigations.

The amount of detail in studies of past contraceptive use varies. The UK Omnibus survey ${ }^{1}$ and the National Survey of Sexual Attitudes and Lifestyles (NATSAL) ${ }^{2}$ ask simply which methods have been used in the last year. The US National Survey of Family Growth (NSFG) ${ }^{3}$ asks about contraceptive method use by month. None of these surveys makes any attempt to check the validity of the respondent's recall.

Accurate recall can be difficult. Prescriber's notes are sometimes used to validate findings ${ }^{4-6}$ but they are often incomplete (or missing) and part of the notes themselves relies on the woman's recall of past contraceptive use. Data from typical primary care notes often lack detail.

Working in a large family planning clinic serving the whole of one Scottish health board area offered the opportunity for a pilot study designed to compare recalled use of contraception over 5 years with detailed information available from standardised clinical case notes.

\section{Methods}

Over 10 clinic sessions the case notes of women with appointments were flagged to identify those who had attended for contraception for the last 5 years or longer.

Dean Terrace Family Planning Clinic, Edinburgh, UK

Anja Katharina Guttinger, MRCOG, MFSRH, Subspecialty Trainee in Sexual and Reproductive Health

Eric Zhong Chen, BSc, MSc, Doctoral Researcher

Correspondence to: Dr Anja Guttinger, The Sandyford Initiative, 2/6 Sandyford Place, Glasgow G3 7NB, UK.

E-mail: aguttinger@nhs.net

\section{Key message points}

- Women can reliably recall use of contraceptive methods to the year of starting and stopping.

- Some women are unable to distinguish use of a combined pill from that of a progestogen-only pill and use of an intrauterine device from the intrauterine system.

Women were approached while waiting to be seen in the clinic and asked to participate in a pilot study comparing their memory of contraceptive use over the past 5 years with the information recorded in their case notes. Women obtaining contraception from other sources [general practitioner (GP), voluntary sector clinic] and those relying on male or female sterilisation were excluded. Data were collected via face-to-face interview by one researcher (AKG) using a standard proforma. Women were asked to recall all methods used including brand names and start/stop dates. Women were prompted to differentiate between combined oral contraceptive (COC) and progestogen-only (POP) pills and the intrauterine system (IUS) from copper intrauterine devices (IUDs). Prompts required to facilitate recall (e.g. "Was there any significant event then?" "Yes, I got married") were documented. A tray displaying commonly used pills (both in and out of their packaging) assisted with recall of brand names if necessary. Finally, the interviewer and respondent constructed a table linking significant life events (such as childbirth, starting college, etc.) with contraceptive history.

Each woman gave verbal consent allowing the doctor to review her case notes. The prescriber's notes and the recall proforma were later compared and the results were collated anonymously and coded for analysis. For this reason, ethics committee approval was not sought for this study.

\section{Data analysis}

An episode of contraceptive use was defined based on the time (in months) from starting one method until discontinuation, or initiation of another method. 
Table 1 Number of contraceptive methods women used in the year prior to interview and for the last 5 years of use

\begin{tabular}{|c|c|c|}
\hline \multirow{2}{*}{$\begin{array}{l}\text { Methods used } \\
\text { during time } \\
\text { frame }(n)\end{array}$} & \multicolumn{2}{|l|}{ Women $(n=30)$} \\
\hline & $\begin{array}{l}\text { During the last } \\
\text { year of use }\end{array}$ & $\begin{array}{l}\text { During the last } \\
5 \text { years of use }\end{array}$ \\
\hline 1 & 22 & 6 \\
\hline 2 & 6 & 11 \\
\hline 3 & 1 & 8 \\
\hline 4 & 1 & 4 \\
\hline 5 & 0 & 1 \\
\hline
\end{tabular}

Disagreement (the difference between the prescriber's notes and the woman's recall) for each episode of contraception used was calculated for the method used, brand name, start/stop dates and duration of use. The recall period for start/stop dates was calculated from the difference between the month of interview and the start/stop month of each episode recorded in the case notes.

Episodes starting before 5 years from the time of interview were excluded from analysis of the start date and duration of use. Use of a method that was ongoing at the time of the interview was excluded in analysis of the stop date. The accuracy of women's recall compared to prescriber's notes for total duration of contraceptive use was measured using Spearman's correlation coefficient.

\section{Results}

Thirty-one women agreed to participate and completed the interview; one was subsequently excluded as she was also seeing her GP for contraceptive advice. All case notes were available for analysis. The mean age of respondents was 35.3 (range, 20-50; standard deviation, 8.0) years and almost half the women $(n=14)$ had completed university education.

During the year prior to interview, 22 women used only one method of contraception, six had used two methods and two women had used three or more methods (Table 1). Over 5 years, six (20\%) women used only one method, 11 (37\%) used two methods and $13(43 \%)$ used three or more.

A total of 73 episodes of different contraceptive method use included no method (2); withdrawal (1); condom (10); Depo-Provera ${ }^{\circledR},(3)$; Implanon ${ }^{\circledR}(7)$; intrauterine methods (12); and oral contraceptives (38). Of the 73 episodes, 47 were included in the analysis of the start date and duration

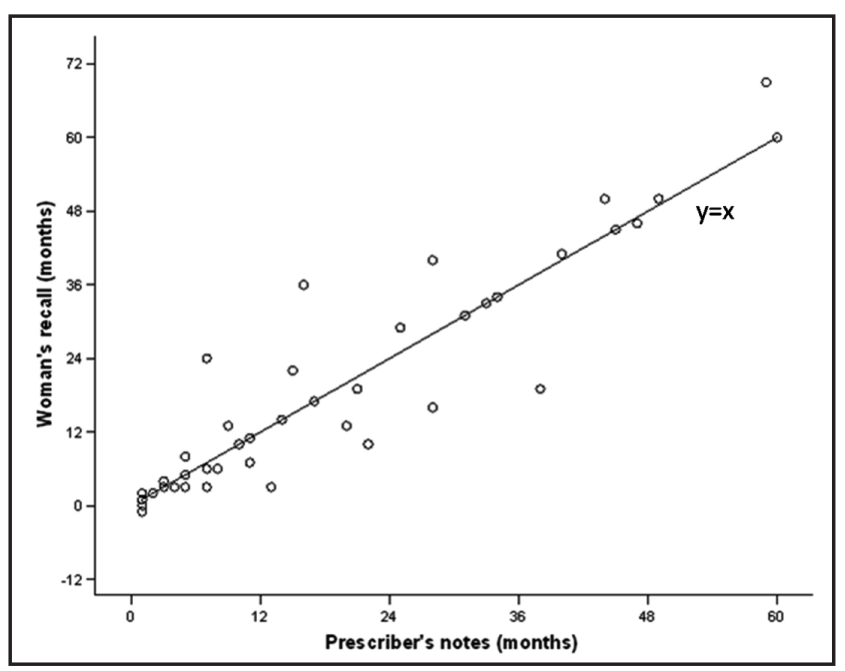

Figure 1 Comparison of the total duration of contraceptive use between woman's recall and prescriber's notes for 47 episodes of contraceptive use of use; a start date was not documented in the case notes for six episodes, and 20 started more than 5 years before the interview. Forty-three episodes were included in the analysis of stop date; 30 episodes ongoing at the time of interview were excluded.

Twenty-two (47\%) episodes of method use were accurately recalled to the month of starting method use; this figure rose to $44(94 \%)$ when episodes with disagreement within \pm 12 months of starting the method were also considered. Similarly, 19 (44\%) and 39 (91\%) episodes were accurately recalled to the month and within \pm 12 months of stopping method use, respectively. Comparing the total duration of use between woman's recall and prescriber's notes (Figure 1), the Spearman's correlation coefficient was $0.94(p<0.01)$ for the 47 episodes analysed. For duration of method use, 18 (38\%) episodes were recalled accurately to within 1 month; when disagreement within \pm 12 months was considered, this figure rose to $44(94 \%)$ episodes. Of the 38 episodes of oral contraceptive use, in $20(53 \%)$ cases women were able to correctly identify the specific brand without prompting. In the remaining 18 episodes, 16 women required prompting to differentiate correctly between COC and POP use. Twothirds (8) of the 12 intrauterine method users were able to distinguish IUS and IUD correctly.

\section{Discussion}

This study was undertaken to determine how accurately women recall contraceptive use. The sample is too small to allow detailed statistical analysis but does provide some interesting findings. We found that women were able to recall accurately the contraceptive methods they had used during the past year. This is reassuring for surveys like NATSAL and Omnibus. ${ }^{1,2}$ In contrast, recall of less than half the episodes of past contraceptive use was accurate to within 1 month, suggesting that for studies aiming to explore patterns of contraceptive switching (particularly frequent switching) a prospective design is advisable. For epidemiological studies, such as those investigating the effect of oral contraceptives on cancer risk, it is important to recognise that the use of $\mathrm{COC}$ and POP cannot be distinguished in 2/38 (7\%) episodes of past oral contraceptive use, even after prompting. Also, researchers and clinicians should be aware that one-third of women (well-educated women moreover) using intrauterine contraception can not distinguish between the IUD and IUS. For clinicians it is reassuring to know that when they ask women which contraceptives they have used in the last year the reply will be reasonably accurate but they may find linking contraceptive use to significant life events useful when trying to obtain a more precise contraceptive history.

There are some caveats. This was a pilot study and, by chance, the women participating were highly educated. Our findings are similar to those described in the OxfordFamily Planning Association study ${ }^{7}$ but that cohort of women was also highly selected. Importantly, since the pilot study was not designed to evaluate the comparisons, we cannot know whether the 'in-depth' interview with a life table approach is measurably superior to simply asking standard survey-style questions. Detailed interviewing takes time and it is important to know whether the time invested is worth the cost involved.

\section{Acknowledgement}

The authors want to thank the women who participated for their help with the study.

Statements on funding and competing interests Funding None identified.

Competing interests None identified. 
References

1 Taylor T, Keyse L, Bryant A. Contraception and Sexual Health 2005/06 (Omnibus Survey Report No. 30). London, UK: Office for National Statistics, 2006.

2 Johnson AM, Wadsworth J, Wellings K, Field J. Sexual Attitudes and Lifestyles. Oxford, UK: Blackwell Scientific Press, 1994; 1-34.

3 Kost K, Singh S, Vaughan B, Trussell J, Bankole A. Estimates of contraceptive failure from the 2002 National Survey of Family Growth. Contraception 2008; 78: 85

4 Harlow SD, Linet MS. Agreement between questionnaire data and medical records. The evidence for accuracy of recall. $A m \mathrm{~J}$
Epidemiol 1989; 129: 233-248.

5 Nischan P, Ebeling K, Thomas DB, Hirsch U. Comparison of recalled and validated oral contraceptive histories. $A m \mathrm{~J}$ Epidemiol 1993; 138: 697-703.

6 van Leeuwen FE, van Duijn CM, Camps MH, Kempers BA, Mentjens MF, Mulder HB, et al. Agreement between oral contraceptive users and prescribers: implications for casecontrol studies. Contraception 1992; 45: 399-408.

7 Coulter A, Vessey M, McPherson K, Crossley B. The ability of women to recall their oral contraceptive histories. Contraception 1986; 33: 127-137.

\section{VIEW FROM PRIMARY CARE}

\section{Popping in for a quickie}

\section{Amos Tavwon}

Another day, another pile of post dropped onto my desk by a smirking member of staff discharging her responsibility and adding to mine. No doubt I'm going to find the usual " $\mathrm{Mr} \mathrm{X}$ invites you to visit his spanking new private rooms to see the marvellous futuristic therapies that poor old you on the NHS haven't a chance in hell of ever providing, over a glass of wine that may have a fancy label but won't be any of the good stuff from his personal cellar", and of course the standard "Pharma X invites you to an evening meeting where much as we'd like to take you to a Michelin-rated restaurant and get you pissed on champagne we've actually struggled to get approval for the IUD-shaped pen that you'll only be given once you've endured our 'rep' at your practice".

Imagine my delight then when from one envelope out pops a toy, game, puzzle, call it what you will. It grabbed my attention though. A plastic ring attached by a cord to a disc-shaped belt hook. Intrigued? I most certainly was. Pull the ring, let go and back against the belt hook it snaps. This is fun. Well it was for a minute or so. Must be some sort of executive stress management toy? That'll come in handy. What else does it do? No sounds. No lights. Getting a bit bored now. Paperwork says it's a CVR. Oh, silly me. Of course I should have known it's a CVR, not to be confused with the Honda CRV, which you certainly can't clip to a belt. Good Lord, that's amazing, a compact video recorder and - I kid you not - the ring, which must be the tape, is only 2 inches in diameter, and the belt-clip recorder itself is only 1 inch in diameter. Incredible. Now, let's see how I record something? Presumably to playback you just pull the ring like we've always done with talking dolls. Pull the string out of their back and off they go: "I love you", "Prepare for action", "Let's do it", "We have an emergency situation".

Nope. Nothing. Ah, the leaflet announces it's a combined vaginal ring containing contraceptive hormones. Clever idea. So let's guess, how do you use it? Clip it onto your belt obviously, like we did a few years ago with personal alcohol gel dispensers so they were ready and easy to use for tackling MRSA. With this CVR, presumably if you find yourself in the need of a 'quickie' or the 'mood de l'amore' takes over but you've no condom available, from a prevention of pregnancy (not STIs obviously) point of view you can pull the cord and either slip the ring into the vagina, or onto the penis, and 'whey hey', off you go!

J Fam Plann Reprod Health Care 2009; 35(3): 175

Ringing in the Changes Surgery, Snapback on the Mount, UK Amos Tavwon, MRcGP, General Practitioner

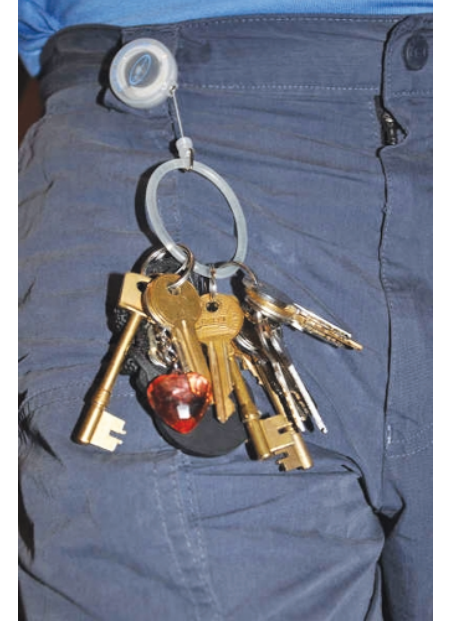

Figure 1 "Out on the pull"

Not quite right. It says here the woman puts it inside the vagina and leaves it for 3 weeks. Fair enough, be prepared and all that. But surely that cord is going to chafe, isn't it? It's going to be like a thong rubbing away on the private parts. Worse still, it could be like having a cheese wire down there. What if the ring springs back out when a woman is walking? I know that happened when a colleague of mine was in the supermarket with a couple of vaginal love balls in situ. She couldn't decide which was more embarrassing: the clicking as she walked when they were where they should be, or trying to explain to the security guard that she hadn't been shoplifting eggs when they fell out. Yes, it says expulsion can occur after a bowel movement, or valsalva. It can come out during intercourse too. I wonder if this means it simply comes out or gets transferred onto the man's penis - from contraception to penile love ring in one easy thrust. Thinking about it, there could potentially be an additional contraceptive choice here as having this ring spring out and snap against the penis might deflate the situation somewhat.

Hang about. The picture on the leaflet doesn't have the belt clip, just the ring itself. So the one I have here is just a demonstration model. Good idea having it attached so someone doesn't walk off with it. I mean, they say doctors are the worst, but patients will lift anything. We've had to chain the chairs in the waiting room. Shame this thing doesn't have a retractor button like some dog leads have.

I should have followed my own advice and read all this before speculating. The real thing is just the ring. OK, now I understand. So into the bin with it? No, I have just the perfect use for it (Figure 1). I wonder just how many keys it will take... 